\title{
Erratum: A New Nonlinear Dynamical System That Leads to Eigenvalues
}

Japan J. Indust. Appl. Math., 9(1992), 133-139

\section{YOSHIMASA NAKAMURA}

Department of Mathematics, Gifu University, Yanagido, Gifu 501-11, Japan

Proposition 2 (p.136) states that two types of fixed points occur on the flow of the dynamical system (6). One is the case of unstable fixed points where $L(\infty)=$ $\lim _{t \rightarrow \infty} L(t)$ has equal diagonal elements as (12-1). The other is that of stable fixed points. $L(\infty)$ takes the form of diagonal matrices as (12-2) and the function (9) has a global minimum on it.

The third type of fixed points should be added to Proposition 2. If $L(\infty)$ is not diagonal but, under a suitable change in basis, the diagonal (or, principal) blocks of $L(\infty)$ are matrices such as (12-1) or (12-2) and any elements of off-diagonal blocks are equal to zero, then $L(\infty)$ is also an unstable fixed point. Here we present an expression of them for $n=3$

$$
L(\infty)=\left(\begin{array}{ccc}
\ell_{11}^{\infty} & \ell_{12}^{\infty} & \ell_{13}^{\infty} \\
\ell_{21}^{\infty} & \ell_{11}^{\infty} & \ell_{23}^{\infty} \\
\ell_{31}^{\infty} & \ell_{32}^{\infty} & \ell_{11}^{\infty}
\end{array}\right), \quad\left(\begin{array}{ccc}
\ell_{11}^{\infty} & 0 & 0 \\
0 & \ell_{22}^{\infty} & 0 \\
0 & 0 & \ell_{33}^{\infty}
\end{array}\right), \quad\left(\begin{array}{ccc}
\ell_{11}^{\infty} & \ell_{12}^{\infty} & 0 \\
\ell_{21}^{\infty} & \ell_{11}^{\infty} & 0 \\
0 & 0 & \ell_{33}^{\infty}
\end{array}\right)
$$

